\title{
Finite Element Analysis of the Model of Mechanical Behavior of SUS420 Steel Scraping Blade in Printing Engineering
}

\author{
Ö. KARAÇALI* \\ Istanbul University, Department of Mechanical Engineering, Avcllar, Istanbul, 34320 Turkey
}

\begin{abstract}
The scraping blade is a critical device for maintaining the uniform thickness of an anilox-developing roll and for removing the excess of ink, water and contamination from the smooth non-engraved portions of the image carrier by controlling the pressure on the developing roller in the printing machine. Not much research can be seen in the literature, related to the material and the geometrical shape of the scraping blade. Due to this fact, a novel simulation method was implemented in ANSYS to control the mechanical behavior of scraping blade and of the anilox rolls. Numerical simulation was carried out using the method of finite element analysis for analysis of the system of SUS420-chrome-containing martensitic stainless steel blade. The purpose of this study was to develop a model of structural behavior to minimize excessive stresses and wears and to achieve an optimal design of the scraping SUS420 stainless steel blade. As a design optimization tool, the finite element analysis was engaged to perform static analysis of scraping structures, scraping blade holders and blades. Flexural deflection analysis and structure optimal design methodology were developed to improve the blade life span. According to the outcomes of this paper, the scraping blade was improved after optimization of the wall thickness and of the tip angle. Thus the smooth scraping quality was improved. These results suggest that a new design with the new blade tip could be beneficial for designers and manufacturers.
\end{abstract}

DOI: 10.12693/APhysPolA.134.10

PACS/topics: finite element analysis, SUS420-stainless steel scraping blade, mechanical behavior

\section{Introduction}

The scraping blades are brought into contact with the anilox rollers while cleaning the surface of the anilox rollers of the excess ink, as shown in Fig. 1. The blade tip is inclined to the point of contact around the rotation axis [1]. As a result of compression and friction, the scraping blade requires to be renewed regularly by abrasion. The cost of anilox rolls is often very high; however, the price of scraper blades is small [2].

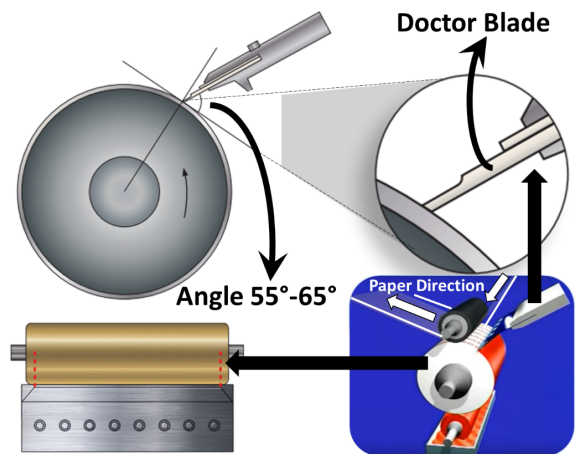

Fig. 1. Research focus: "scraping blade" under investigation, shown in a printing machine.

The scraping blades are considered to wear out because of the intention to preserve constant ink metering for distribution for the duration of the print cycle [3]. Since

\footnotetext{
*e-mail: ozdogank@istanbul.edu.tr
}

the scraping blade scrapes (or meters) away the extra ink from the anilox roll, it then coats a specific film of ink onto a printing roller. The contact pressure between the developing roller and the scraping blade is one of the significant factors for image quality and durability of anilox rolls [4]. The scraping blades, the materials they are made of and their usage in the paper machines are complex issues to comprehend due to surface stress, roughness of the wearing surfaces, life expectancy and fatigue failure of the subsurface [5].

Though the earlier studies [6] mentioned above had primarily focused on analyzing the consequences of the cleaning blade on purging performance, in this research the material properties, the shape and the load parameters were investigated for improving the scraping performance. The main research problems of the study reported in this article are summarized in Fig. 2. Based on the experimental data, in this study the stress value of von-Mises criteria was established by calculating the amount of distortions, deformations and deflections, as explained in the next section.

\section{Materials and methods}

The finite element analysis (FEA) was used to discover the scraping blade angle and shape constraints, which minimize the von-Mises value whilst fulfilling the design limitations for supporting the right contact between the scraping blade and the anilox roll. A parametric FEA model was created for the structural analysis procedure and to determine the amount of distortion. Today, FEA method is applied in modeling of the mechanical behavior of materials and is used to determine the process constraints $[7-9]$. The functioning parameters, integrated in 


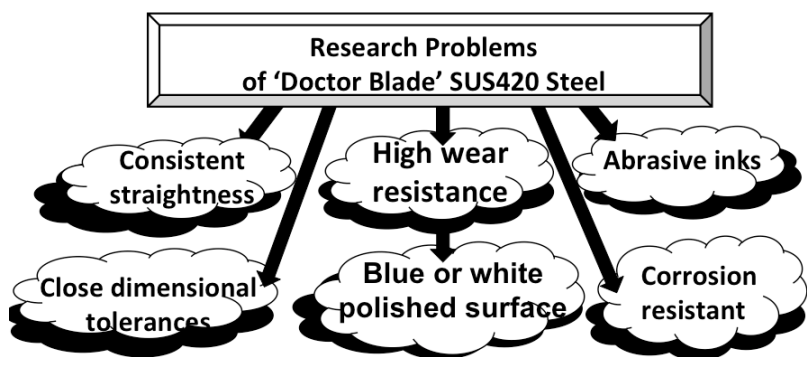

Fig. 2. Research area and material characteristics of scraping blade made of SUS420 steel.

design limitations for a required scheme were calculated by mechanical evaluation of the scraping blade using ANSYS software (version 18.00).

The research has emphasized that shear stress plays a vital part within the printing machinery of gravure offset line printing. For this research, a printing model displaying the shear stress reliance on the photogravure position and angle was used, as shown in Fig. 3.

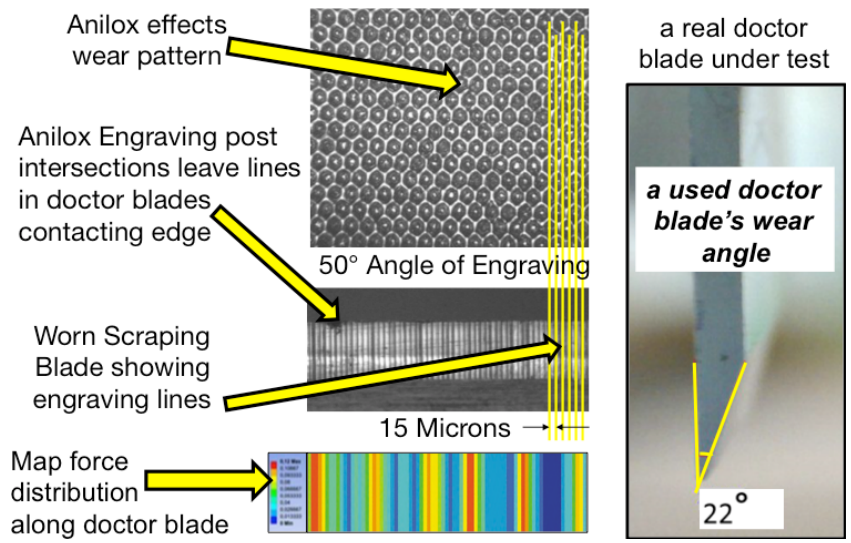

Fig. 3. Anilox effects wear pattern and worn scraping blade showing engraving lines.

The decrease of the inner angle between the printing path and the pattern-line route increases the shear pressure, thus improving the quantity of transported ink in the off stage. Printing analysis utilizing pattern-line widths of $81 \mu \mathrm{m}$ and $20 \mu \mathrm{m}$ indicates the point reliance of the accordance width, thickness and total transferred ink, reflecting the impact of the shear stress. The impact of the internal perspective on cross-sectional differences of strains with a width of $20 \mu \mathrm{m}$ and with perspective variation is more than that in strains with a width of $81 \mu \mathrm{m}$, which agrees with the theoretical assessment that shear stress has an additional effect on thinner lines, as demonstrated in Fig. 3. The solid correlation between the experimental data and the theoretical model backs the confirmation of the theoretical model. Scraping blades were amassed from industrial printing operations and the cross sectional evaluation was carried out to evaluate the scraping blade tips.
These outcomes indicate the quantity of the wear and from this, the real cleaning angle may be attained. This information then was utilized within a rearranged numerical model with the intention to assess the contact between the anilox roll and the scraping blade. The aftermaths display each deflection of the blade and the sensitivity of the device to adjustments inside the scraping blade load. A parametric analysis was carried out on the scraping blade by taking into account the blade and the process set-up. This model was defined due to small amount of loading and boundary conditions. For both loading conditions, an encastre boundary condition was applied at the surface of the fixed end of the blade.

To simulate an end load, a surface traction load was applied to the surface at the free end of the blade. For uniform loading, a uniform pressure was applied to the top surface of the entire blade. Overall, the performance of each element was fully integrated quadratic element with two elements along the length of the blade. In this case, the mesh is really too coarse to make a fair judgment. Figure 4 displays the tip displacement vs. mesh density for the loaded scraping blade.

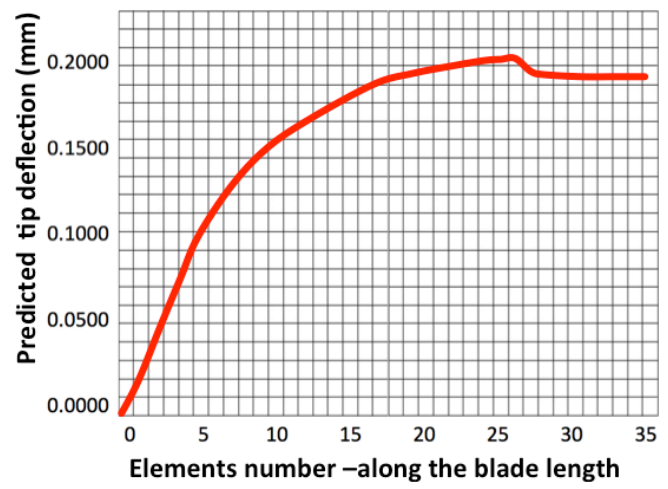

Fig. 4. Loaded scraping blade: tip displacement vs. mesh density.

Figure 5 presents the main model simulation of scraping blade shape for evaluation of the structure under static and dynamic loads, concluding the total deformation and von Mises stress distribution. This modeling helped us to increase the strength of the design. ANSYS is a specific purpose commercial software package based on LS-DYNA module and the finite element analysis. At the scraping blade tip the von-Mises stress arising due to geometrical shape design was greatly modified compared to that of the preliminary optimized shape.

Equivalent stresses von Mises criteria under different contact forces were studied on scraping blade under investigation. The contact forces in scraping blades were calculated by data fitting method. However, the contact forces on blade were maximized. As the result, a more rigid assembly of scraping blades may provide a tighter contact that might move the ink smoothly on the surface of the blade. The contact forces in the scraping blades were computed and are displayed in Fig. 6. 


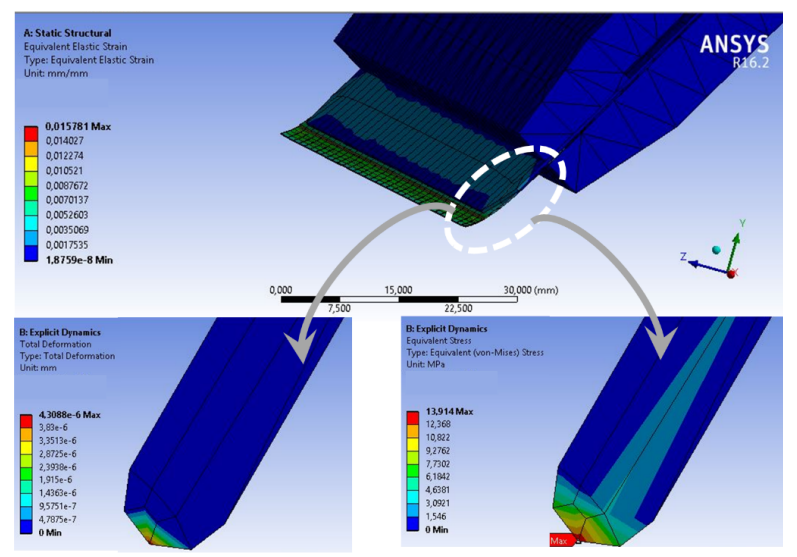

Fig. 5. Total deformation and von Mises stress distribution in scraping blade.

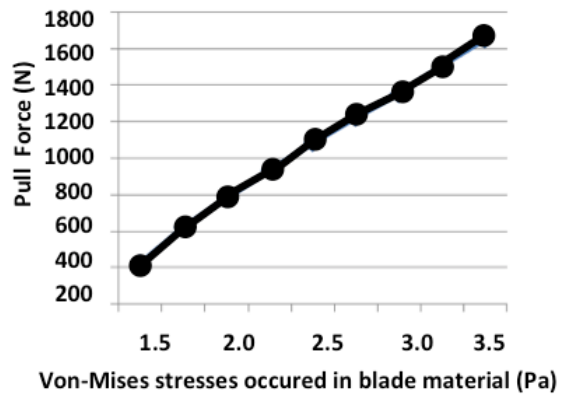

Fig. 6. Equivalent von Mises stresses.

The developed model required a particular computation to minimize $\sigma_{\max }$ at the greatest stress of von-Mises criteria for the scraping blade prototype. First the nonlinearity of the properties of SUS420 material was evaluated. Afterwards, the mesh model for the finite element method was created taking into account the modification in design parameters. The structural analysis was performed latter. Then, the improved durability of the critical blade angle was computed as 21 degrees in terms of breakage and abrasion of the blade due to maximum von-Mises and compressive stresses at the lowest level.

\section{Experimental results and discussion}

In this study a design problem for scraping blade was outlined to analyze von-Mises stress while fulfilling the structure restrictions for providing optimal contact between the scraping blade and the anilox roll. ASTM1095W.Nr.-1.1274 and SS 1870 (Fatigue strength and wear resistance international standards) were taken into consideration for the experimental set up in the simulation. In mechanical model the contact pressure between the blade tip and the rolls is the physical parameter measured by operators.

The scraping performance of the system was optimized to achieve an increase of the blade lifecycle by modeling the contact between the scraping blade and the anilox roll using ANSYS software. Experimental results were found to be in a good agreement with our calculations. When the length of blade body was $640 \mathrm{~mm}$, it was found that for a blade width of $1.9 \mathrm{~mm}$ and for $1.6 \mathrm{~mm}$ deflection the thickness of the film remaining after the surface scraping is about $25 \mu \mathrm{m}$. The contact nip length was $28 \mu \mathrm{m}$, while the maximum conjunction pressure was found to be $8 \mathrm{MPa}$ and $3.8 \mathrm{MPa}$ under static and elastohydrodynamic conditions, respectively. According to the outcomes of this investigation, the scraping blade was improved after the optimization of the wall thickness. Thus the smooth scraping quality was improved.

\section{Conclusions}

Using the proposed design methodology, the maximum von Mises stress was reduced by $36 \%$ compared to the initial design, while fulfilling all design limitations. To verify the rationality of the design calculation, a scraping blade was manufactured according to the optimal design solution. The correct setup of the blade was carried out and printer operation was examined. In the test $47 \%$ more sheets were processed without the pollution of the charge roller and no picture tainting after this research analyses was observed. The results of the verification test achieved in the experiments that were run repeatedly undoubtedly prove the validity of the design methodology by FEA. These results suggest that the new design with the new blade tip could be useful for printing engineers and blade producers.

\section{Acknowledgments}

This work was supported by Scientific Research Projects Coordination Unit of Istanbul University. Project number 28251.

\section{References}

[1] K. Suganuma, Printing Technology. In: Introduction to Printed Electronics. SpringerBriefs in Electrical and Computer Engineering 74, Springer, New York, 2014.

[2] R. Kitsomboonloha, S.J.S. Morris, X. Rong, V. Subramanian, Langmuir 28(48), 16711 (2012).

[3] G. Kono, Y. Inagaki, H. Yabuno, T. Nohara, M. Kasama, J. Comput. Nonlinear Dynam. 7(1), 011006 (2011).

[4] C. Park, J. Lee, J. Jeong, D. Choi, Struct. Multidisc. Optim. 49, 131 (2014).

[5] D. Jiang, D. Shu, J. of Mater.Eng. and Perform. 18, 255 (2009).

[6] M . Kasama, M. Yoshizawa, Y. Yu, T. Itoh, Y. Itoh, J. Syst. Des. Dyn. 2(3), 849 (2008).

[7] Ö. Karaçalı, A.T. Ülgüel, Acta Phys. Pol. A 125(2), 183 (2014).

[8] Ö. Karaçalı, D. Çetiner, Acta Phys. Pol. A 125(2), 180 (2014).

[9] Ö. Karaçalı, Acta Phys. Pol. A 127(4), 1195 (2015). 